\title{
Real-World Impact of Retrievable Stents for Acute Ischemic Stroke on Disability Utilizing the National Inpatient Sample
}

\author{
Anit Behera $^{\mathrm{a}, \mathrm{b}} \quad$ Eric Adjei Boakye ${ }^{\mathrm{c}, \mathrm{d}} \quad$ Jahnavi Trivedi ${ }^{\mathrm{b}} \quad$ Eric Armbrecht ${ }^{\mathrm{a}}$ \\ Amer Alshekhlee ${ }^{e}$ Randall Edgell ${ }^{f}$ \\ a St. Louis University Center for Health Outcomes Research, St. Louis, MO, USA; bSt. Louis \\ University School of Medicine, St. Louis, MO, USA; 'CSouthern Illinois University School of \\ Medicine, Department of Population Science and Policy, Springfield, IL, USA; d Southern \\ Illinois University School of Medicine, Department of Internal Medicine, Springfield, IL, USA; \\ eSSM Health DePaul Hospital, Bridgeton, MO, USA; ${ }^{f}$ St. Louis University School of Medicine, \\ Department of Neurology, St. Louis, MO, USA
}

\section{Keywords}

Retrievable stent $\cdot$ Acute ischemic stroke $\cdot$ National inpatient sample $\cdot$ Mechanical thrombectomy $\cdot$ In-hospital mortality

\begin{abstract}
Purpose: We assess the impact of retrievable stent (RS) compared to first-generation devices on in-hospital mortality and disability in patients with acute ischemic stroke (AIS). Methods: Using the National Inpatient Sample, data were obtained for patients with a primary diagnosis of AIS who underwent mechanical thrombectomy (MT) and were admitted to US hospitals between 2010 and 2014. Two time periods were compared: 2010-2012 (pre-RS Food and Drug Administration [FDA] approval) and 2013-2014 (post-RS FDA approval). Disability level was used to classify outcomes as minimal disability, moderate to severe disability, or in-hospital mortality. Weighted, multivariable logistic regression was used to assess the association between MT device type and disability. Results: A total of 2,443,713 weighted patients admitted with AIS were identified; 148,923 (4.9\%) of these received intravenous tissue plasminogen activator; and 23,719 (0.8\%) underwent MT. In multivariable logistic regression analysis, the odds of in-hospital mortality decreased (OR $0.69,95 \% \mathrm{Cl} 0.59-0.82)$ in the post-RS time-period compared with pre-RS time. The odds of moderate-to-severe disability decreased (OR 0.88 , $95 \% \mathrm{Cl} 0.73-1.06)$ compared with minimal disability. In-hospital mortality rates decreased successively over the 4 years in the MT-treated patients $(p<0.001)$. Conclusions: The FDA approval of RS technology after 2012 was associated with decreased in-hospital mortality when compared with the 3-year interval prior. These findings provide an indication that the RCT data on the efficacy of RS technology are translating into improved real-world outcomes.
\end{abstract}


Behera et al.: Real-World Impact of RS Technology

\section{Introduction}

Until recently, intravenous tissue plasminogen activator (IV-tPA) used within $4.5 \mathrm{~h}$ was the only standard-of-care treatment option for patients with acute ischemic stroke (AIS) [1]. While mechanical thrombectomy (MT) has long held promise for the treatment AIS, firstgeneration devices introduced in 2004 and 2008 were not shown to be superior to IV-tPA alone in randomized controlled trials (RCT) [2-4]. Food and Drug Administration (FDA) approval of the Solitaire Revascularization device (Medtronic, Dublin, Ireland) and the TREVO ProVue Retriever (Stryker, Kalamazoo, MI, USA) in 2012 added retrievable stent (RS) technology to available MT devices. This technology has since largely replaced first-generation devices due to the results of multiple RCTs showing the efficacy of MT with RS combined with IV-tPA exceeded that of IV-tPA alone [5-9]. MT with RS is now considered a standard of care treatment for patients with large vessel occlusion within a 6-h window [10]. There are ongoing postmarketing registries studying RS technology; however, the published real-world data on RS technology is limited. In the current study, we identified patients treated with MT between 2010 and 2014. This time-period spans the years before and after the introduction and widespread use of RS devices for MT and therefore allowed us to look at outcomes associated with RS FDA approval.

\section{Methods}

Dataset and Patient Demographics

Data were obtained and analyzed from the National Inpatient Sample (NIS) database for the years 2010-2014. The NIS database is publicly available de-identified data that are exempted from institutional review board approval. NIS is the largest all-payer in-patient care database in the United States. Between 2010 through 2014, the NIS data collection was redesigned. Prior to 2012, the NIS represented 100\% of discharges from a random sample of all hospitals (nonfederal hospitals, including those managed by city and state governments and for-profit and not-for-profit organizations) in the United States. In 2012, a new sampling methodology was adopted that included a random sample of discharges from all hospitals participating in the Healthcare Cost and Utilization Project (HCUP). Detailed information on the design of the NIS is available at http://www.hcup-us.ahrq.gov.

The International Classification of Disease - Clinical Modification, 9th revision, (ICD-9 CM) primary diagnosis codes 433.01, 433.11, 433.21, 433.31, 433.81, 433.91, 434.01, 434.11, 434.91, 435.9, 436, 437.1, and 437.9, were used to identify patients with AIS. ICD-9 CM procedure codes were utilized to recognize the patients who were treated with MT and/or IV-tPA. Patients who had MT treatment were identified using the ICD-9 procedure code 39.74, as previously described by Brinjikji et al. [11]. If a patient received ICD-9 CM code 99.10, the patient was also labeled as receiving IV-tPA. Previous investigations have shown these codes to have a true positive rate of $84 \%$ for stroke admissions [12] and a sensitivity of 55\% and specificity of $98 \%$ for thrombolytic use [13].

\section{Measures}

The main independent variable was MT time periods. The time periods were based on the FDA approval of the TREVO ProVue retriever and the Solitaire revascularization device in 2012. Patients who had MT performed in the 2010-2012 time-period were classified as pre-RS and in the 2013-2014 time period were classified as post-RS. Covariates included in the study were selected based on previous literature and theoretical and clinical significance [14-17]. Patient sociodemographic and clinical factors included age, gender, race/ethnicity, median household income based on the individual's zip code for that current year grouped into 4 quartiles as defined by the NIS (patients with an unknown quartile were not included in the analysis), location based on the NCHS Urban-Rural Code, 2006, admission day, admission status, and Elixhauser comorbidity score [18] as determined from the AHRQ comorbidity data files. Secondary intracerebral hemorrhage, a medical comorbidity not included in the Elixhauser score, was identified by ICD-9-CM secondary diagnosis codes: $(430,431,432$, and 432.9). Length of stay in hospital was classified as short stay 
( $\leq 5$ days) or prolonged stay ( $>5$ days). Finally, hospital-level factors that were assessed included geographic region, teaching status, and control/ownership (whether private not-for-profit/investor-owned or government nonfederal).

The main outcomes of interest in this study were (1) in-hospital mortality (dead/alive) and (2) disability level as described in a previous literature categorizing discharge outcome [19]; home discharge was classified as minimal disability and any other discharge status was classified as moderate-to-severe disability for all patients discharged alive.

Statistical Analysis

The Statistical Analysis Software (SAS; version 9.4) was used to perform procedures which incorporate survey sampling weights to account for the complex sampling design used in NIS and to provide nationally representative estimates of the US population (SAS Institute Inc., Cary, NC, USA). Descriptive statistics, mean \pm SD for continuous variables and weighted proportions were used to analyze participants' characteristics. Sociodemographic, clinical- and hospital-level factors were compared by MT device type using chi-square tests for categorical variables and independent samples $t$ test for continuous variables where appropriate.

Multivariable logistic regression analyses were conducted to evaluate the association between MT device type/time periods and the outcome of interests and the association between in-hospital mortality and disability level controlling for covariates. These covariates adjusted for include: age, gender, race/ethnicity, household income, admission day, admission status, Elixhauser comorbidity score, secondary intracerebral hemorrhage, length of stay, hospital geographic region, teaching status, and control/ownership. We performed trend analysis for MT per year and in-hospital mortality with MT-treated patients from 20102014. All analyses were 2 tailed, and statistical significance was determined using $p<0.05$.

\section{Results}

\section{Baseline Demographic and Clinical Characteristics}

A total of 3,036,738 weighted patients admitted with AIS were identified; 148,923 (4.9\%) of these received intravenous tPA; and 23,719 (0.8\%) underwent MT. From 2010 to 2014, the utilization of MT increased from 0.55 to $1.07 \%(p<0.001$; Fig. 1). In-hospital mortality decreased successively over the 5 years (2010-2014) in the MT-treated patients $(p<0.001$; (Fig. 2). Table 1 summarizes the characteristics of patients hospitalized between 2010 and 2014 , overall and stratified by MT time period. Approximately $17 \%$ died in the hospital and $68.1 \%$ of the discharges were moderate-to-severe. Overall, the average age was 66.8 (SD = $15.1)$ yearsand average comorbidity score was $3.6(\mathrm{SD}=1.8)$. A majority of patients were white (64.2\%), admitted on a weekday (74.6\%), not transferred (72.5), and were admitted to teaching hospitals (84.5\%).

Patients within the post-RS period were more likely to be white and black and less likely to be Hispanic and other/unknown $(p=0.0022)$. Elixhauser comorbidity score increased $(p=0.0016)$ in the post-RS period compared to the pre-RS period indicating that sicker patients were being treated in the later time-period. Patients within the pre-RS period were more likely to receive IV-tPA $(p<0.0001)$ and be transferred to a hospital performing the MT procedure $(p<0.0001)$. Patients within the post-RS period were more likely to be admitted to hospitals in the Northeast and South and less likely from the Midwest and West regions $(p<0.001)$. No statistical difference was found between the 2 time periods for age, gender, household income, county location, intracranial hemorrhage, admission day, length of stay, hospital control, and teaching status between the 2 study periods (Table 1).

\section{Clinical Outcomes at Discharge}

Minimal disability and moderate-to-severe disability increased in patients treated during the post-RS era: 13.8 vs. $16.5 \%$ and 67.5 vs. $68.7 \%$ respectively. However, in-hospital mortality 
Fig. 1. National trend percentages in proportion of ischemic stroke patients who received mechanical thrombectomy treatment in 2010-2014 ( $n=612,536$ [unweighted]). Data source: National Inpatient Sample.
Fig. 2. National trend percentages in mortality among ischemic stroke patients who received mechanical thrombectomy treatment in 2010-2014 ( $n=4,758$ [unweighted]). Data source: National Inpatient Sample.
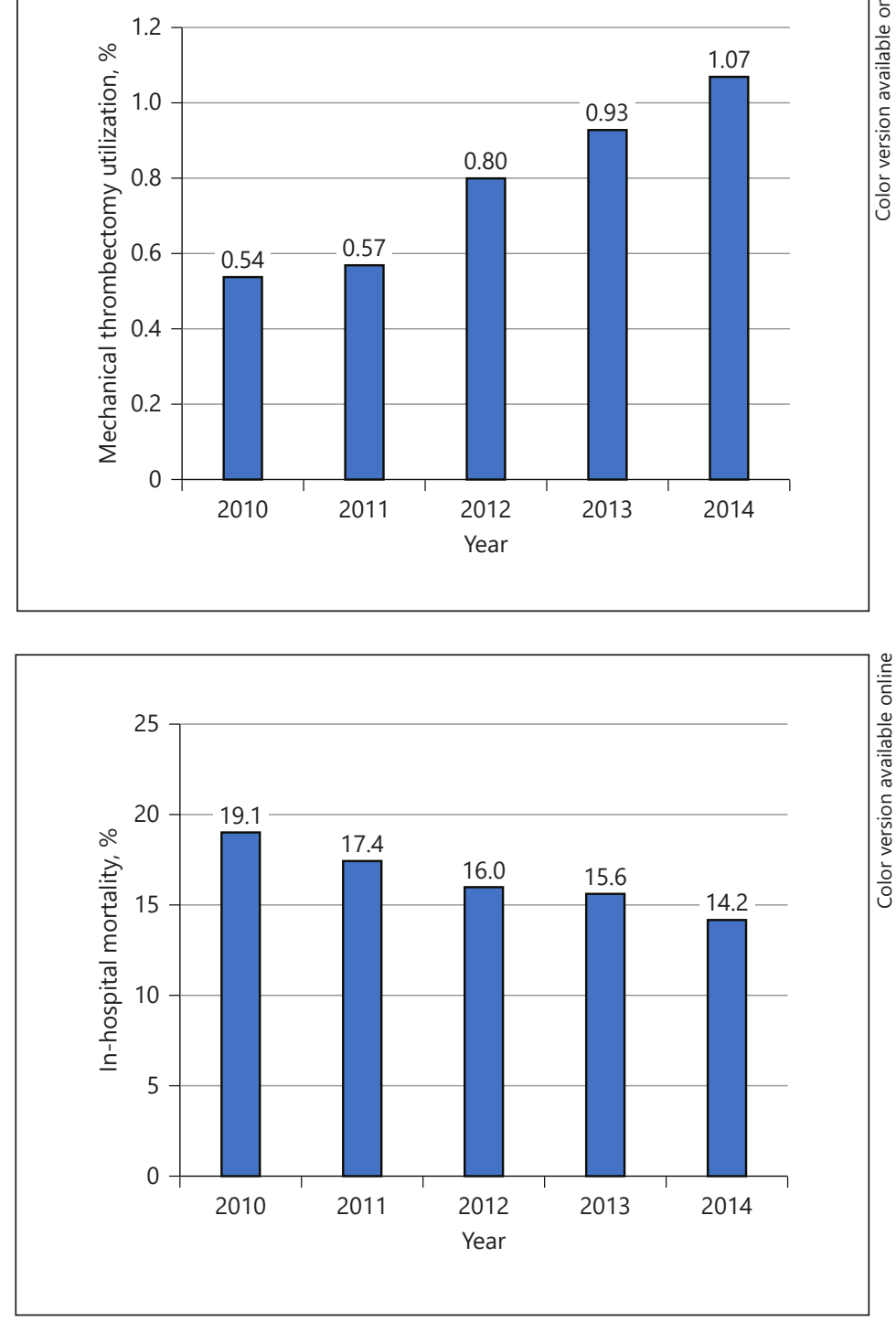

decreased from the pre-RS period to the post-RS period (18.7 vs. $14.8 \%, p=0.0003$, Table 1 ). The decrease in-hospital mortality persisted (OR 0.69, 95\% CI 0.59-0.82) after adjusting for age, gender, race, household income, Elixhauser comorbidity score, length of stay, IV-tPA use, presence of intracerebral hemorrhage, hospital regional location, hospital control, and hospital teaching status in the multivariable logistic regression analysis. Compared with females, the odds of in-hospital mortality were higher among males in-hospital mortality (OR $1.21,95 \%$ CI 1.03-1.42), as it were for patients whose household income fell in the lowest quartile (1st quartile) in-hospital mortality (OR 1.40, 95\% CI 1.10-1.78) compared with the highest quartile (fourth). Patients with a presence of intracerebral hemorrhage had higher odds of in-hospital mortality (OR 2.59, 95\% CI 2.16-3.10; Table 2).

Furthermore, the odds of moderate-to-severe disability were not statistically significant compared with minimum disability, after adjusting for the factors mentioned above in the multivariable logistic regression model (Table 2). However, advanced age (OR 1.04, 95\% CI 1.03-1.05) and comorbidities (OR 1.27, 95\% CI 1.20-1.35) were associated with higher odds 
Table 1. Demographic and clinical characteristics of patients with acute ischemic stroke treated with mechanical thrombectomy between 2010 and 2014

\begin{tabular}{|c|c|c|c|c|}
\hline $\begin{array}{l}\text { Variables } \\
\text { Mean } \pm \text { SD or } w \%\end{array}$ & $\begin{array}{l}\text { Total } \\
(n=23,719)\end{array}$ & $\begin{array}{l}2010-2012 \\
(n=11,859)\end{array}$ & $\begin{array}{l}2013-2014 \\
(n=11,860)\end{array}$ & $p$ value \\
\hline Age, years & $66.8 \pm 15.1$ & $66.4 \pm 15.2$ & $67.2 \pm 14.9$ & 0.0617 \\
\hline Gender & & & & 0.7577 \\
\hline Male & 49.9 & 49.6 & 50.1 & \\
\hline Female & 50.1 & 50.4 & 49.9 & \\
\hline Race & & & & 0.0022 \\
\hline White & 64.2 & 62.7 & 65.6 & \\
\hline Black & 11.8 & 11.7 & 11.8 & \\
\hline Hispanic & 7.2 & 6.7 & 7.7 & \\
\hline Other/unknown & 16.8 & 18.8 & 14.8 & \\
\hline Household income & & & & 0.1891 \\
\hline 1st Quartile & 25.5 & 24.2 & 26.7 & \\
\hline 2nd Quartile & 24.3 & 24.4 & 24.3 & \\
\hline 3rd Quartile & 25.7 & 26.0 & 25.3 & \\
\hline 4th Quartile & 24.5 & 25.4 & 23.6 & \\
\hline County & & & & 0.1086 \\
\hline$>1,000,000$ population & 62.0 & 63.5 & 60.5 & \\
\hline$<1,000,000$ population & 23.5 & 22.5 & 24.4 & \\
\hline Other/unknown & 14.5 & 13.9 & 15.1 & \\
\hline Elixhauser comorbidity score & $3.6 \pm 1.8$ & $3.5 \pm 1.8$ & $3.7 \pm 1.9$ & 0.0016 \\
\hline Admission day & & & & 0.4003 \\
\hline Weekday & 74.6 & 75.1 & 74.0 & \\
\hline Weekend & 25.4 & 24.9 & 26.0 & \\
\hline Admission status & & & & $<0.0001$ \\
\hline Not transferred & 72.5 & 69.9 & 75.1 & \\
\hline Transferred & 27.5 & 30.1 & 24.9 & \\
\hline Length of stay & & & & 0.0958 \\
\hline Short stay & 40.1 & 38.9 & 41.3 & \\
\hline Prolonged stay & 59.9 & 61.1 & 58.7 & \\
\hline IV-tPA use (yes) & 49.4 & 54.1 & 44.6 & $<0.0001$ \\
\hline Intracerebral hemorrhage (yes) & 21.5 & 21.9 & 21.2 & 0.5280 \\
\hline Hospital regional location & & & & $<0.0001$ \\
\hline Northeast & 18.8 & 18.9 & 18.8 & \\
\hline Midwest & 25.4 & 27.3 & 23.6 & \\
\hline South & 32.0 & 27.9 & 36.1 & \\
\hline West & 23.8 & 26.0 & 21.5 & \\
\hline Hospital control & & & & 0.2239 \\
\hline Government, nonfederal & 15.4 & 14.7 & 16.0 & \\
\hline Private, not-profit & 84.6 & 85.3 & 84.0 & \\
\hline Hospital teaching status & & & & 0.0009 \\
\hline Non-teaching & 15.5 & 17.2 & 13.7 & \\
\hline Teaching & 84.5 & 82.8 & 86.3 & \\
\hline Disability & & & & 0.0003 \\
\hline Minimal & 15.2 & 13.8 & 16.5 & \\
\hline Moderate-to-severe & 68.1 & 67.5 & 68.7 & \\
\hline In-hospital mortality & 16.8 & 18.7 & 14.8 & \\
\hline
\end{tabular}

SD, standard deviation; IV-tPA, intravenous tissue plasminogen activator; w\%, weighted percentage. Data source: national inpatient sample. 
Interventional

Neurology

Table 2. Multivariate logistic regression: predictors of in-hospital mortality and moderate-to-severe disability of patients with acute ischemic stroke treated with mechanical thrombectomy between 2010 and 2014

\begin{tabular}{|c|c|}
\hline Intervent Neurol 2019;8:60 & \\
\hline DOI: $10.1159 / 000495160$ & $\begin{array}{l}\text { (c) } 2018 \text { S. Karger AG, Basel } \\
\text { www.karger.com/ine }\end{array}$ \\
\hline
\end{tabular}

\begin{tabular}{|c|c|c|}
\hline Variables & $\begin{array}{l}\text { In-hospital } \\
\text { mortality } \\
\text { aOR }(95 \% \mathrm{CI})\end{array}$ & $\begin{array}{l}\text { Disability } \\
\text { (alive only) } \\
\text { aOR (95\% CI) }\end{array}$ \\
\hline \multicolumn{3}{|l|}{ MT device type } \\
\hline 2010-2012 & referent & referent \\
\hline 2013-2014 & $0.69(0.59-0.82)$ & $0.88(0.73-1.06)$ \\
\hline Age, years & $1.02(1.01-1.03)$ & $1.04(1.03-1.05)$ \\
\hline \multicolumn{3}{|l|}{ Gender } \\
\hline Male & $1.21(1.03-1.42)$ & $0.88(0.74-1.06)$ \\
\hline Female & referent & referent \\
\hline \multicolumn{3}{|l|}{ Race } \\
\hline White & referent & referent \\
\hline Black & $1.02(0.78-1.35)$ & $1.06(0.79-1.43)$ \\
\hline Hispanic & $1.06(0.77-1.47)$ & $0.64(0.44-0.91)$ \\
\hline Other/unknown & $1.06(0.84-1.32)$ & $0.89(0.69-1.15)$ \\
\hline \multicolumn{3}{|l|}{ Household income } \\
\hline 1st Quartile & $1.40(1.10-1.78)$ & $1.17(0.89-1.54)$ \\
\hline 2nd Quartile & $1.12(0.88-1.42)$ & $1.01(0.77-1.31)$ \\
\hline 3rd Quartile & $1.18(0.94-1.48)$ & $1.04(0.81-1.34)$ \\
\hline 4th Quartile & referent & referent \\
\hline Elixhauser comorbidity score & $1.03(0.98-1.08)$ & $1.27(1.20-1.35)$ \\
\hline \multicolumn{3}{|l|}{ Admission day } \\
\hline Weekday & referent & referent \\
\hline Weekend & $1.14(0.95-1.37)$ & $1.31(1.06-1.63)$ \\
\hline \multicolumn{3}{|l|}{ Admission status } \\
\hline Not transferred & $\mathrm{N} / \mathrm{A}$ & referent \\
\hline Transferred & $\mathrm{N} / \mathrm{A}$ & $1.53(1.22-1.93)$ \\
\hline \multicolumn{3}{|l|}{ Length of stay } \\
\hline Short stay & referent & referent \\
\hline Prolonged stay & $0.27(0.23-0.32)$ & $4.21(3.46-5.13)$ \\
\hline \multicolumn{3}{|l|}{ IV tPA } \\
\hline Yes & referent & referent \\
\hline No & $1.17(0.99-1.38)$ & $1.19(0.99-1.44)$ \\
\hline \multicolumn{3}{|l|}{ Intracerebral hemorrhage } \\
\hline No & referent & referent \\
\hline Yes & $2.59(2.16-3.10)$ & $1.94(1.46-2.59)$ \\
\hline \multicolumn{3}{|l|}{ Hospital regional location } \\
\hline Northeast & $1.34(1.04-1.71)$ & $1.57(1.15-2.15)$ \\
\hline South & $1.16(0.92-1.48)$ & $0.79(0.62-1.02)$ \\
\hline West & $1.11(0.87-1.42)$ & $0.94(0.72-1.22)$ \\
\hline Midwest & referent & referent \\
\hline \multicolumn{3}{|l|}{ Hospital control } \\
\hline Government, nonfederal & 1.17 (0.93-1.47) & $0.92(0.71-1.19)$ \\
\hline Private & referent & referent \\
\hline \multicolumn{3}{|l|}{ Hospital teaching status } \\
\hline Teaching & referent & referent \\
\hline Non-teaching & $1.08(0.86-1.36)$ & $1.22(0.94-1.59)$ \\
\hline
\end{tabular}

aOR, adjusted OR; IV-tPA, intravenous tissue plasminogen activator. Data source: national inpatient sample.

of moderate-to-severe disability as were prolonged length of stay (OR 4.21, 95\% CI 3.465.13) compared with short length of stay. Other factors associated with higher odds of moderate-to-severe disability were admission on a weekend (OR 1.31, 95\% CI 1.06-1.63) versus weekday, and having a presence of intracerebral hemorrhage (OR 1.94, 95\% CI 1.462.59; Table 2). 


\section{Discussion}

The findings from our study should give added confidence that the results of the landmark RCT of MT published in 2015 [5-9] translate to the real world. We found that the number of patients with minimal disability after MT increased in the post-RS period (2010-2012:13.8\% vs. 2013-2014: 16.5\%). Furthermore, we found that in-hospital mortality had decreased from the pre-RS period to the post-RS period (2010-2012: 18.7\% vs. 2013-2014: 14.8\%) and trend analysis revealed that it was decreasing year to year. The first RCT focusing on RS technology was published in 2014: The Multicenter Randomized Clinical Trial of Endovascular Treatment for AIS (MR CLEAN) trial [5]. RS devices were shown to be beneficial with an adjusted OR of 1.67 (95\% CI 1.21-2.30) of functional recovery at 90 days [5]. With the publication of these results, 4 simultaneous RCTs were halted for interim analysis. Ultimately, all 4 trials were ended without completing their planned enrollment due to statistically significant benefit in the MT arm (NNT range 2.8-6.5) [6-9]. Among the recently reported RCTs, only the ESCAPE trial showed a significant reduction in mortality in the MT + IV-tPA group versus the IV-tPA group alone (10.4 vs. 19.0\%, respectively) [6]. However, our comparison groups differ from the RCTs in that we are comparing results of first- and second-generation device periods.

As noted above, the percentage of patients with minimal disability increased from the pre-RS to post-RS time periods. A prior publication by Hassan et al. [20] also examined minimal disability as an outcome in the NIS from 2004 to 2009 for AIS patients treated with first-generation devices. In contrast, they found a decrease in the level of minimal disability where $32.3 \%$ of patients had minimal disability in the 2004-2007 time period (post-MERCI) and $27.2 \%$ of patients had minimal disability in the 2008-2009 time period (post-Penumbra) [20] indicating worse outcomes in the later time period. Our finding of a greater number of patients with minimal disability in the post-RS period is a reassuring trend and is consistent with other evidence of the superiority of second-generation devices.

We also report a decrease in the odds for mortality among patients treated in the post-RS period when compared to patients in the pre-RS period after controlling several co-factors. The decrease in the OR is more notable when one considers the post-RS population was older and had an increased Elixhauser comorbidity index. This also indicates that physicians are becoming more aggressive in utilizing MT. When interpreting these results, several methodological factors should be considered. We have utilized discharge location as a surrogate marker for disability. Discharge location is selected relatively soon after the MT procedure and therefore may not be a reflective medium to long-term disability. In adhering to the previously utilized methodology [19], we combined all non-home discharges in the moderate-tosevere disability category. This includes locations such as acute rehabilitation facilities, which have been known to take mild-to-moderately disabled patients that go on to achieve complete or near-complete recovery at 90 days and beyond.

We found that the utilization of MT increased from 0.55 to $1.07 \%$ from 2010 to 2014 . Hassan et al. [20] documented an increase in utilization from the 2004-2007 time period to the 2008-2009 time period (0.1-0.6\%) indicating a continued trend in the usage of MT. The increase in MT utilization in these patients could be explained by a number of factors including an increasing number of comprehensive stroke centers, an increased perception of efficacy and acceptance by physicians, and a higher diagnosis-related group-based hospital reimbursement for patients undergoing "surgical" procedures. Nevertheless, despite gaining acceptance as a standard-of-care treatment for acute large vessel occlusion, MT remains an underutilized therapy. Between 4 and $14 \%$ of AIS patients are thought to be eligible for MT [21], but our study shows only 1.1\% received MT in 2014. 
While we have identified and analyzed AIS patients treated with MT using previously described methodology, several limitations in the NIS database must be considered. In the second time period, old technology might still have been used, thus not providing a true representation of second-generation RS. Second, the database does not contain standard scales used to measure stroke severity (e.g., National Institutes of Health Stroke Scale) or disability (e.g., modified Rankin scale scores). Because of this, our findings are unable to be translated to a standardized score. In addition, data do not allow us to recognize sub-groups defined by initial severity of deficits, time to treatment, and 90-day outcomes. These factors are known to be associated with stroke outcomes. We are unable to study procedural details for individual patients as this information is not included in the NIS. Finally, our assessment of functional outcome was based on discharge location and this variable may not be an accurate predictor of long-term recovery.

\section{Conclusion}

Our study has utilized the NIS to assess the real-world impact of RS technology approval in 2012. We have found an association between improved clinical outcomes including mortality with the approval of this technology. In addition, there has been a trend toward greater utilization of MT in AIS patients that are older and have a greater number of comorbidities. These findings are consistent with RCT data proving the benefit of MT with RS when added IV-tPA. It also confirms the previous observation of increasing adoption of this treatment outside of clinical trials. However, the procedure remains underutilized compared to the entire population eligible patients. Future studies should include patients treated after publication of the RCT data, as this may have spurred even greater adoption of the technique.

\section{Disclosure Statement}

The authors declare that they have no conflicts of interest to disclose.

\section{References}

1 National Institute of Neurological Disorders and Stroke rt-PA Stroke Study Group. Tissue plasminogen activator for acute ischemic stroke. N Engl J Med. 1995 Dec;333(24):1581-7.

2 Broderick JP, Palesch YY, Demchuk AM, Yeatts SD, Khatri P, Hill MD, et al.; Interventional Management of Stroke (IMS) III Investigators. Endovascular therapy after intravenous t-PA versus t-PA alone for stroke. N Engl J Med. 2013 Mar;368(10):893-903.

3 Kidwell CS, Jahan R, Gornbein J, Alger JR, Nenov V, Ajani Z, et al.; MR RESCUE Investigators. A trial of imaging selection and endovascular treatment for ischemic stroke. N Engl J Med. 2013 Mar;368(10):914-23.

4 Ciccone A, Valvassori L, Nichelatti M, Sgoifo A, Ponzio M, Sterzi R, et al.; SYNTHESIS Expansion Investigators. Endovascular treatment for acute ischemic stroke. N Engl J Med. 2013 Mar;368(10):904-13.

5 Berkhemer OA, Fransen PS, Beumer D, van den Berg LA, Lingsma HF, Yoo AJ, et al.; MR CLEAN Investigators. A randomized trial of intraarterial treatment for acute ischemic stroke. N Engl J Med. 2015 Jan;372(1):11-20.

6 Goyal M, Demchuk AM, Menon BK, Eesa M, Rempel JL, Thornton J, et al.; ESCAPE Trial Investigators. Randomized assessment of rapid endovascular treatment of ischemic stroke. N Engl J Med. 2015 Mar;372(11):1019-30.

7 Saver JL, Goyal M, Bonafe A, Diener HC, Levy EI, Pereira VM, et al.; SWIFT PRIME Investigators. Stent-retriever thrombectomy after intravenous t-PA vs. t-PA alone in stroke. N Engl J Med. 2015 Jun;372(24):2285-95.

8 Campbell BC, Mitchell PJ, Kleinig TJ, Dewey HM, Churilov L, Yassi N, et al.; EXTEND-IA Investigators. Endovascular therapy for ischemic stroke with perfusion-imaging selection. N Engl J Med. 2015 Mar;372(11):1009-18.

9 Jovin TG, Chamorro A, Cobo E, de Miquel MA, Molina CA, Rovira A, et al.; REVASCAT Trial Investigators. Thrombectomy within 8 hours after symptom onset in ischemic stroke. N Engl J Med. 2015 Jun;372(24):2296-306. 
10 Powers WJ, Derdeyn CP, Biller J, Coffey CS, Hoh BL, Jauch EC, et al.; American Heart Association Stroke Council. 2015 american heart association/american stroke association focused update of the 2013 guidelines for the early management of patients with acute ischemic stroke regarding endovascular treatment: A guideline for healthcare professionals from the american heart association/american stroke association. Stroke. 2015 Oct; 46(10):3020-35.

11 Brinjikji W, Rabinstein AA, Kallmes DF, Cloft HJ. Patient outcomes with endovascular embolectomy therapy for acute ischemic stroke: a study of the national inpatient sample: 2006 to 2008. Stroke. 2011 Jun;42(6): 1648-52.

12 Broderick J, Brott T, Kothari R, Miller R, Khoury J, Pancioli A, et al. The Greater Cincinnati/Northern Kentucky Stroke Study: preliminary first-ever and total incidence rates of stroke among blacks. Stroke. 1998 Feb;29(2): 415-21.

13 Qureshi AI, Harris-Lane P, Siddiqi F, Kirmani JF. International classification of diseases and current procedural terminology codes underestimated thrombolytic use for ischemic stroke. J Clin Epidemiol. 2006 Aug;59(8): 856-8.

14 Villwock MR, Padalino DJ, Deshaies EM. Trends in mortality following mechanical thrombectomy for the treatment of acute ischemic stroke in the USA. J Neurointerv Surg. 2016 May;8(5):457-60.

15 Villwock MR, Singla A, Padalino DJ, Deshaies EM. Acute ischaemic stroke outcomes following mechanical thrombectomy in the elderly versus their younger counterpart: a retrospective cohort study. BMJ Open. 2014 Mar; 4(3):e004480.

16 Sonig A, Lin N, Krishna C, Natarajan SK, Mokin M, Hopkins LN, et al. Impact of transfer status on hospitalization cost and discharge disposition for acute ischemic stroke across the US. J Neurosurg. 2016 May; 124(5):122837.

17 Saad A, Adil MM, Patel V, Owada K, Winningham MJ, Nahab F. Clinical outcomes after thrombectomy for acute ischemic stroke on weekends versus weekdays. J Stroke Cerebrovasc Dis. 2014 Nov-Dec;23(10):2708-13.

18 Elixhauser A, Steiner C, Harris DR, Coffey RM. Comorbidity measures for use with administrative data. Med Care. 1998 Jan;36(1):8-27.

19 Qureshi AI, Chaudhry SA, Hassan AE, Zacharatos H, Rodriguez GJ, Suri MF, et al. Thrombolytic treatment of patients with acute ischemic stroke related to underlying arterial dissection in the United States. Arch Neurol. 2011 Dec;68(12):1536-42.

20 Hassan AE, Chaudhry SA, Grigoryan M, Tekle WG, Qureshi AI. National trends in utilization and outcomes of endovascular treatment of acute ischemic stroke patients in the mechanical thrombectomy era. Stroke. 2012 Nov;43(11):3012-7.

21 Palaniswami M, Yan B. Mechanical thrombectomy is now the gold standard for acute ischemic stroke: implications for routine clinical practice. Intervent Neurol. 2015 0ct;4(1-2):18-29. 